\title{
OBSERVATIONS OF EUV RECURRING JETS IN AN ACTIVE REGION CONFINED BY CORONAL LOOPS
}

\author{
YAn-Fang Zheng ${ }^{1,2}$, Feng WAng ${ }^{3}$, Kai FAn Ji ${ }^{3}$, And Hui Deng ${ }^{3}$ \\ ${ }^{1}$ National Astronomical Observatories, Yunnan Astronomical Observatory, Chinese Academy of Sciences, \\ P. O. Box 110, 650011, Kunming, China \\ E-mail : zhengyf@ynao.ac.cn \\ ${ }^{2}$ University of Chinese Academy of Sciences, Beijing 100049, China \\ 3 Computer Technology Application Key Lab of Yunnan Province, Kunming University of Science and \\ Technology, Kunming, Yunnan 650500, China \\ E-mail : cnwangfeng@gmail.com,dh@cnlab.net and jkf@cnlab.net \\ (Received May 14, 2013; Accepted August 12, 2013)
}

\begin{abstract}
Recurring jets, which are jets ejected from the same site, are a peculiar type among various solar jet phenomena. We report such recurring jets ejecting from the same site above an active region on January 22, 2012 with high-resolution multi-wavelength observations from Solar Dynamics Observatory(SDO). We found that the recurring jets had velocities, lengths and lifetimes, but had similar directions. The visible brightening appeared at the jet base before each jet erupted. All the plasma produced by the recurring jets could not overcome the large coronal loops. It seemed that the plasma ejecting from the jet base was confined and guided by preexisting coronal loops, but their directions were not along the paths of the loops. Two of the jets formed crossing structures with the same preexisting filament. We also examined the photospheric magnetic field at the jet base, and observed a visible flux emergence, convergence and cancellation. The four recurring jets all were associated with the impulsive cancellation between two opposite polarities occurring at the jet base during each eruption. In addition, we suggest that the fluxes, flowing out of the active region, might supply the energy for the recurring jets by examining the SDO/Helioseismic and Magnetic Imager (HMI) successive images. The observational results support the magnetic reconnection model of jets.
\end{abstract}

Key words : Sun: jets — Sun: filament — Sun: magnetic fields — Sun: coronal loops

\section{INTRODUCTION}

Jet as a special catalog of dynamical phenomena in the solar atmosphere is often associated with flares or flare-brightening, and has been studied at various wavelengths: $\mathrm{H} \alpha$ surges, EUV jets, and X-ray jets (Jiang et al. 2007; Chen et al. 2009; Shimojo et al. 1996). It is widely accepted that jets are associated with certain photospheric magnetic features, such as emerging magnetic flux (Liu \& Kurokawa 2004), converging magnetic systems (Canfield et al. 1996), and evolving(increasing or decreasing) magnetic flux regions (Shimojo et al. 1998). Magnetic emergence and cancellation between opposite polarity flux were often found to occur at the jet base in the photosphere (Chae et al. 1999; Jiang et al. 2007). A model of jets, indicating that the jets were produced by magnetic reconnection (Shibata et al. 1992), not only is widely held by researchers (Patsourakos et al. 2008; Pariat et al. 2009, 2010), but also supported by observational results.

In recent years, observations of jets often focused on the relationship between jets and their concomitant

Corresponding Author: Y. F. Zheng phenomenon or/and photospheric magnetic field. Some previous observations showed that mini-filament eruptions are correlated with EUV or X-ray jets and $\mathrm{H} \alpha$ surges. Chae et al. (1999) first noticed that a small erupting filament was followed by EUV jet and $\mathrm{H} \alpha$ surge, and it was associated with a micro-flare. Some recent observations indeed showed that mini-filament eruptions on the quiet Sun and active region can lead to blowout EUV jet (Hong et al. 2011; Shen et al. 2012). A few observations showed that jets can be affected by other magnetic structures. Jiang et al. (2008) reported an observation of direct magnetic interactions between a transequatorial jet and interconnecting loops. Some recurrent jets, which occurred at an active region, were reported by Wang et al. (2002) and Chifor et al. (2008). Yang et al. (2011) confirmed that the recurring jets were consistent with a model, which implied that magnetic reconnection between emerging magnetic flux and ambient magnetic field occurred in the lower atmosphere. A blowout surge, which was ejected by the eruption of a small filament and was confined by large coronal loops was reported by Yang et al. (2012). These observations suggest that a careful study of jets with attention to concomitant phenomena will provide clues 


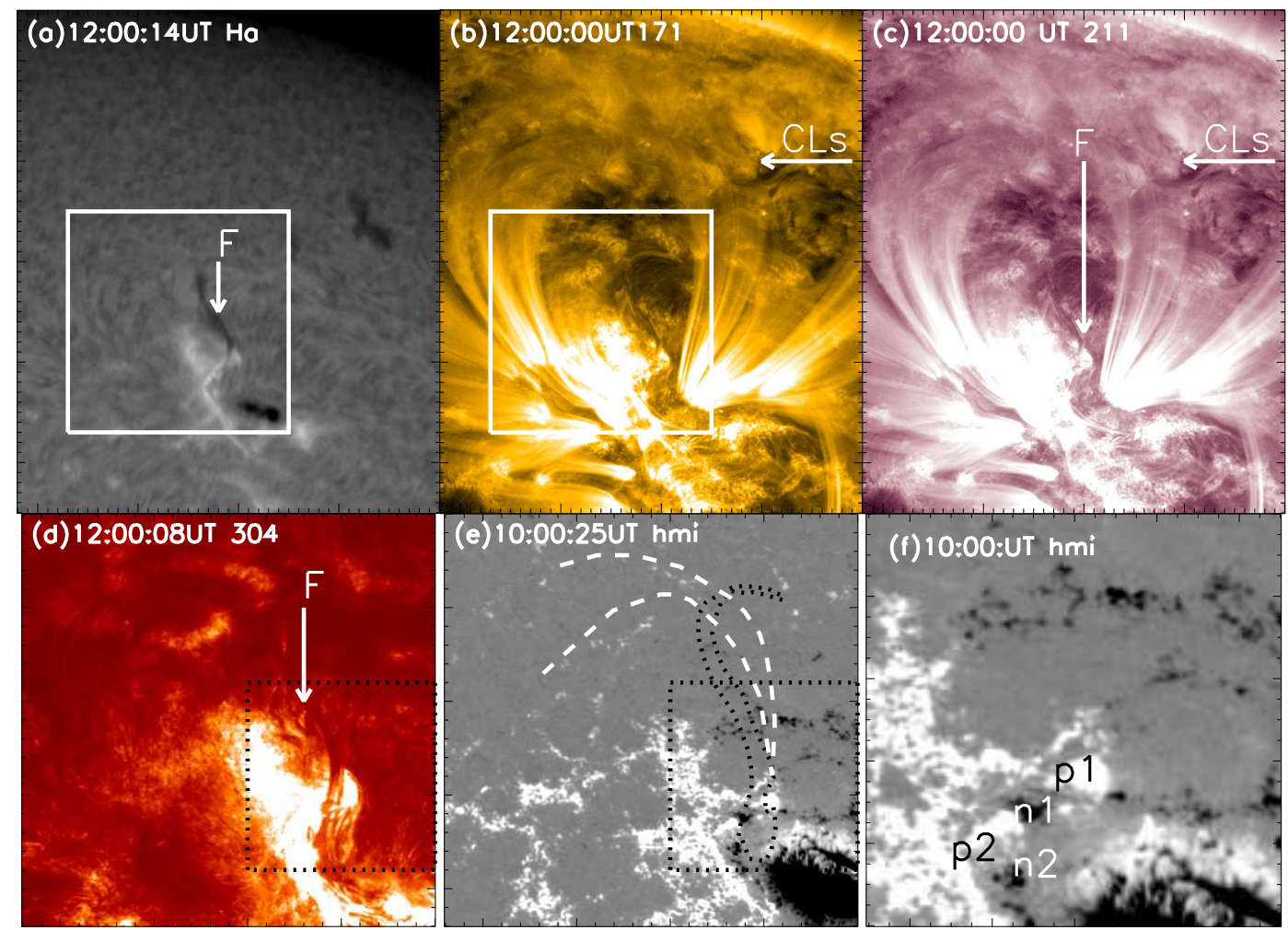

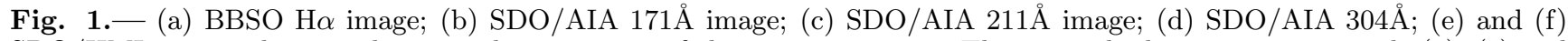
SDO/HMI images showing the general appearance of the eruptive region. The vertical white arrows in panels (a), (c) and (d) indicate the filament located at the jet base, and the aclinic white arrows in panels (b) and (c) indicate the coronal loops. The field of view (FOV) of panels (a) - (c) is $420^{\prime \prime} \times 500^{\prime \prime}$. The FOV of (d) and (e), indicated by the white boxes in panels (a) and (b), is $220^{\prime \prime} \times 220^{\prime \prime}$. The FOV of (f), indicated by the black dots boxes in panels (d) and (e), is $100^{\prime \prime} \times 100^{\prime \prime}$. The black dotted curves and white dotted curves indicate the profiles of filament and one of the recurring jets respectively.

to understand their origins and dynamics.

On January 22, 2012, four recurring jets, which ejected from the edge of AR 11402, were confined by large coronal loops. As a result, the jets formed a crossing structure with a preexisting filament. This gave us an opportunity to investigate the physical characteristics of recurring jets and their concomitant phenomenon. In this paper, we present multi-wavelength observations of the event, and show the evolutions of the jets and their photospheric magnetic field.

\section{INSTRUMENT AND DATA}

The observations used in the present study include:

1. The full-disk $\mathrm{H} \alpha$ center images, which were obtained from the BBSO, New Jersey Institute of Technology, USA, were used to interpret the general appearance of the footpoints of the recurring jets. The $\mathrm{H} \alpha$ observations have a cadence of 1 minute and a pixel width of $1^{\prime \prime}$.

2. Full-disk EUV images from SDO/Atmospheric Imaging Assembly(AIA) (Lemen et al. 2012), which obtains $171 \AA, 304 \AA$, and $211 \AA$ images have high cadences of up to $12 \mathrm{~s}$ and short exposures of $0.1-2 \mathrm{~s}$ with a pixel width of $0.6^{\prime \prime}$.

3. Full-disk line-of-sight magnetograms from SDO/HMI (Schou et al. 2012) at a 45 s cadence with a precision of $10 \mathrm{G}$, and the pixel width is the same with SDO/AIA.

\section{RESULTS}

We examined the recurring jets ejecting from the northwest of NOAA AR 11402(N29W24) (indicated by the white box in Fig. 1a) on January 22, 2012. Fig. 1 shows the general appearance of the jet base on $\mathrm{H} \alpha$ image, SDO/AIA images and their location on the magnetograms before the eruptions. The NOAA AR 11402 had a very complex configuration, and was connected with the NOAA AR 11401(N17W25). Large coronal loops and an S-shaped filament can be identified on SDO $171 \AA$ and $211 \AA$ images (see Fig. 1b,c). The white boxes in panels (a) and (b) indicate the FOV of Fig. 2 , involved by the large coronal loops. The profiles of the filament and jet measured from the SDO/AIA 304 $\AA$ image at 12:57UT are overlaid in panel (e). For convenience, the jet base is outlined by black dotted box in Fig. 1d and e, and the flux regions of opposite polarities are marked as "p1" , "p2", "n1", and "n2" (see 


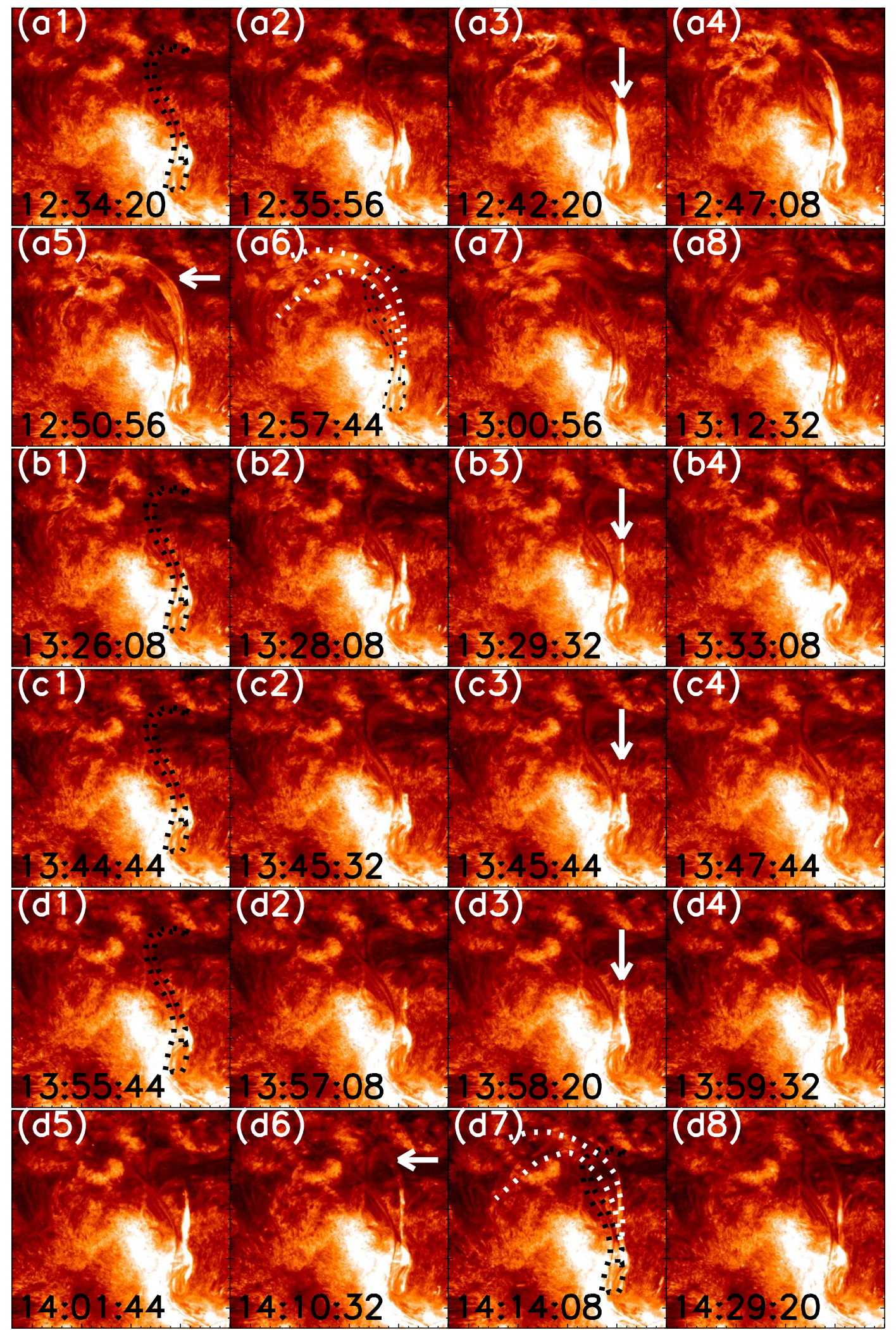

Fig. 2.- Time sequences of SDO/AIA $304 \AA$ images show the four recurring jets. (a1)-(a8) show the evolutions of the first jet; (b1)-(b4) show the second jet; (c1)-(c4) show the third jet; (d1)-(d8) show the fourth jet. The FOV, indicated by the white boxes in Fig. 1, is $220^{\prime \prime} \times 220^{\prime \prime}$. 


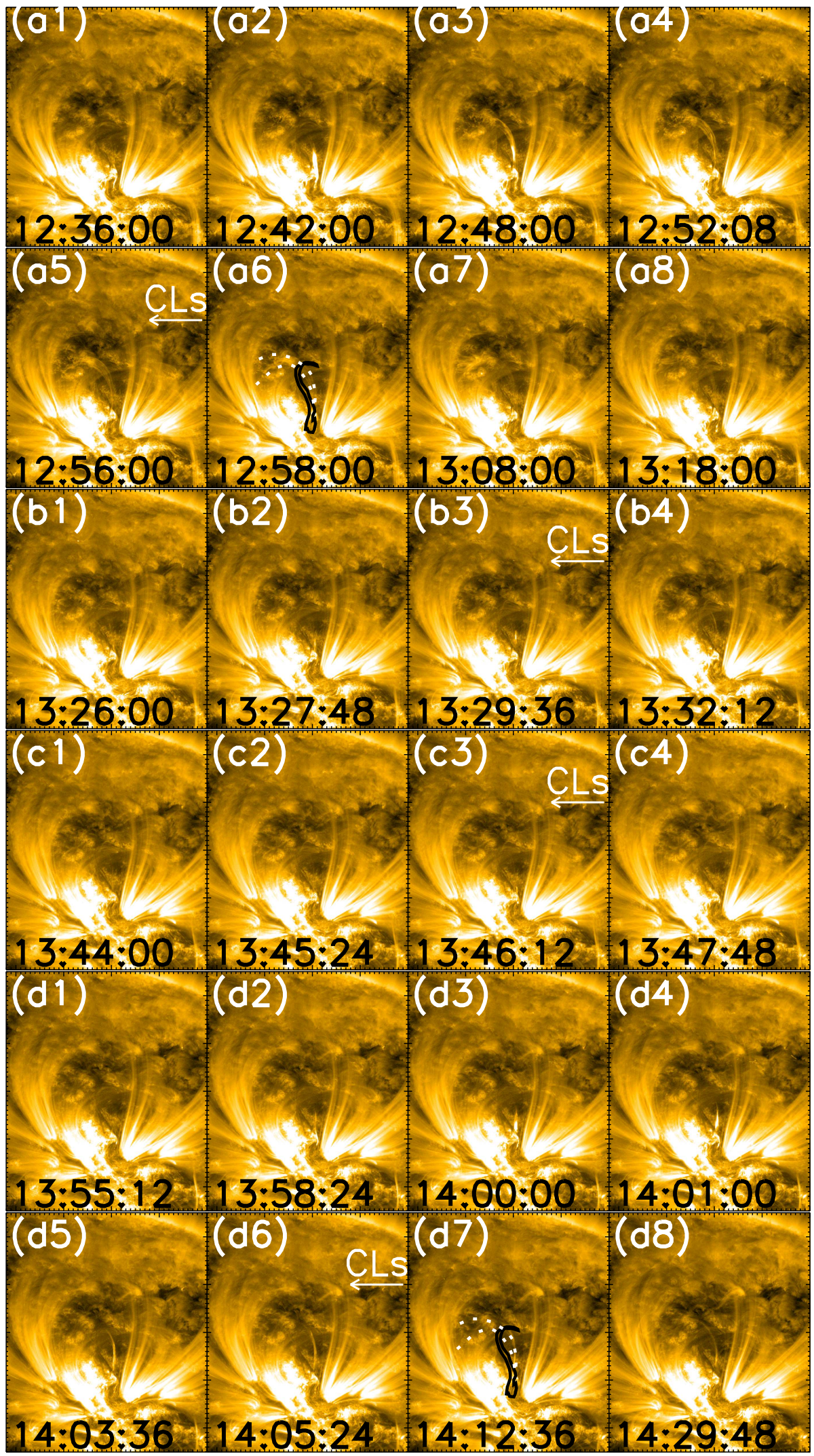

Fig. 3. - The black dotted curves and white dotted curves indicate the profiles of filament and one of the recurring jets respectively. Time sequences of SDO/AIA $171 \AA$ images show the relationship between the four recurring jets and coronal loops. (a1)-(a8) show the evolutions of the first jet. (b1)-(b4) show the second jet. (c1)-(c4) show the third jet. (d1)-(d8) show the fourth jet. The FOV is $420^{\prime \prime} \times 500^{\prime \prime}$. 

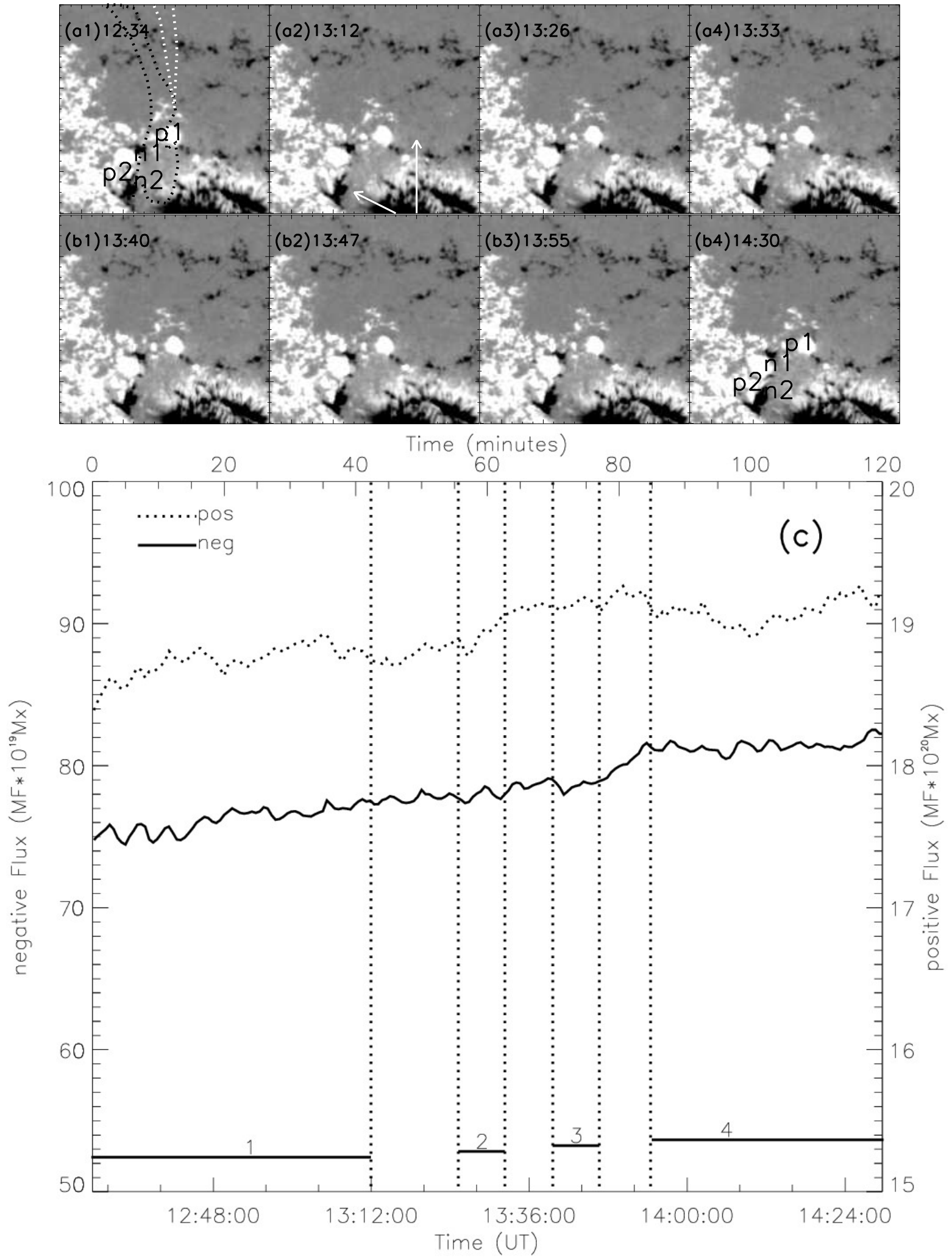

Fig. 4. - Time sequences of SDO/HMI magnetograms show the base of the four recurring jets. (a1)-(a4) and (b1)-(b4) show magnetic filed of the jet base. The FOV is $100^{\prime \prime} \times 100^{\prime \prime}$. Panel (c) shows the positive (dotted lines) and negative (solid lines) magnetic fluxes within the panel (a) and (b) during the four recurring jets,of which the absolute value of positive is scaled by y-axis on the right. 
Fig. 1f).

The recurring jets are covered by SDO/AIA $304 \AA$ and $171 \AA$ direct images. Four EUV jets, which ejected from the same jet base, are presented in Figs. 2 and 3. The S-shaped profiles indicated the preexisting Sshaped filament in the active region (see Fig. 2; a1, b1, c1, and d1). Figs. 2a and 3a show the evolutions of the first jet. The jet base located at one footpoint of the filament brightened at 12:34:20 UT. It stretched to the north shaped like a spire (see Fig. 2; a3) and strode the filament (see Fig. 2; a4-a7). A crossing structure formed during the process, and its profiles are overlaid in Fig. 2; a6. This jet lasted for about $38 \mathrm{~min}$, and achieved its maximum length of $160 \mathrm{~mm}$ at 12:50:56UT. We estimated its velocity to be around $161 \mathrm{~km} / \mathrm{s}$. The $171 \AA$ images show that the base of the jets also involves a coronal loop. The plasma, ejecting from the jet base and falling into another end of coronal loops, was seemed to be confined and guided by large coronal loops (see Fig. 3; a3-a6). The second jet, which started around 13:26UT, was much fainter than the first. It lasted for around $7 \mathrm{~min}$, and achieved a maximum length of $43 \mathrm{~mm}$ at 13:29:32 UT, quickly faded away and completely vanished at 13:33UT (see Fig. 2b). The velocity was around $213 \mathrm{~km} / \mathrm{s}$. The plasma produced by the second jet seemed to rise along the path of the first jet. From $171 \AA$ images (see Fig. $3 \mathrm{~b}$ ), we found that the second jet also exhibited brightening at the jet base. The second jet rose along the same path as the first jet, but was too weak to reach the other end of the coronal loop. The evolutions of the third jet resembled that of the second and are shown in Figs. 2c and 3c. This event lasted for less than 5 min, achieved its maximum length of $19 \mathrm{~mm}$ at 13:45:44 UT, quickly faded away and completely vanished at 13:47:44UT. The plasma ejected from the base of the jet rose rapidly along the path of the first jet and we estimated its velocity to be around $326 \mathrm{~km} / \mathrm{s}$. A brightening phenomenon at the jet base was also seen in the event. In Figs. 2d and 3d, we see a fourth jet ejected from the active region at 13:55:44UT. This jet lasted for about $35 \mathrm{~min}$, and achieved a maximum length of $160 \mathrm{Mm}$ at 14:14:08 UT. We estimate the velocity of the event to be $147 \mathrm{~km} / \mathrm{s}$. We found that the plasma ejected from the jet base traced along the first jet's plasma and strode the preexisting filament, and a crossing structure formed (indicated by the profiles in panel $\mathrm{d} 7)$.

By comparing the four recurrent jets, we found that the plasma produced by the four eruptions not only ejected from the same jet base, but also rose along the same path (indicated by vertical white arrows in Fig. 2). This plasma was too weak to overcome the coronal loops. We also saw that the preexisting filament appeared very weak brightening by inspecting the SDO/AIA successive images and movies. All the jets in present study were related with the jet base brightening and coronal loops (indicated by horizontal white arrows in Fig. 3). It is possible that the first and fourth jets represent the ejections. Typical crossing structures formed during the two eruptions. The plasma ejected from the base of the jet, in the two cases, strode the preexisting filament and formed a crossing structure with it (indicated by the horizontal white arrows in Fig. 2). The plasma subsequently fell at the other end of the concomitant coronal loop.

By means of the SDO/HMI line-of-sight magnetograms (see Fig. 4a,b), we investigated the evolutions of the photospheric magnetic fields in the active region. The profiles of filament and the jet measured from SDO/AIA image at 12:57UT are overlaid in panel (a1). The black dotted curves indicate the filament and the white dotted curves indicate the jet. For conveniences, the positive and negative fluxes in the magnetic fields of the jet base were marked as "p1", "p2", "n1", and "n2" (see Fig. 4; a1). It is easy to see that the positive fluxes flow out of the negative area (indicated by the white arrows in Fig. 4; a2). Fluxes were found to emerge from the base of jet. We see that p1 and $n 1$ areas move farther apart while $\mathrm{p} 2$ and $\mathrm{n} 2$ move nearer eventually merging at 13:58 UT. It is also obvious that the p2 and n3 areas increased in size continuously until their merger, whereas $\mathrm{p} 1$ and $\mathrm{n} 1$ areas decreased. The flux changes in the area, completely containing the positive and the negative patches are plotted in Fig. 4c. We divided the changing of fluxes into four stages: $12: 30-13: 12 \mathrm{UT}$ (the first jet), $13: 26-13: 33 \mathrm{UT}$ (the second jet), 13:40-13:47UT (the third jet), and 13:55-14:30UT (the fourth jet). These stages are corresponding to the four jet eruptions. In the first stage, the positive flux decreased (increased) while the negative flux increased (decreased). Such changing flux patterns suggest the emergence of magnetic flux and the cancellation between opposite polarities. In the second stage, positive flux decreased at the beginning (before 13:28UT), but then increased quickly to a higher value. On the other hand, the negative flux first showed rapid increase during the period of positive fluxes increase, but the increased flux then reduced to the initial level. The changing of magnetic fluxes during the second jet eruption indicated the emerging of positive flux and the cancellation between the two opposite polarities. The scenario during the eruption of the third jet was similar to the second stage. Before the fourth jet eruption, we can see that the negative flux underwent a rapid increase. On the other hand, the positive flux first increased (before 13:50), but then decreased to the lower level. In the fourth stage, the negative showed a roughly little increase, while the positive flux first decreased, but then increased to a higher level. These changes of fluxes indicated that the impulsive cancellation between the opposite polarities occurred during the fourth jet. 


\section{CONCLUSION AND DISCUSSION}

Recurrent jets, located at the edge of a complex active region (AR11402), were examined by using of $\mathrm{H} \alpha$, EUV $(171 \AA, 304 \AA)$ and photospheric magnetic field observations. Our main observational results are summarized in the following points.

(1)These jets are ejected from the same site. The plasma produced by jet eruptions had similar directions. They reached a maximum projected height of from $160 \mathrm{~mm}$ to $19 \mathrm{~mm}$. they had a transverse velocity of $147-326 \mathrm{~km} / \mathrm{s}$ and lasted from a few to tens of minutes.

(2)Flare-like brightening appeared at the jet base before the jet eruption. A pre-existing filament at the base of jet brightened slightly during the eruptions. The plasma produced by the first and the fourth jets formed crossing structures with the preexisting filament.

(3) The magnetic field of the recurring jets located at the mixed polarity section at the edge of an active region. The magnetic emergence, convergence and cancellation were clearly found during their eruptions. It is indicated that the flux, which continuously flowed from the active region, could supply the energy for the recurring jets.

In this study, the recurring jets had very different velocities, lengths and lifetimes, but they were very similar in directions and were correlated well with the emergence, convergence and cancellation of photospheric magnetic flux. It suggests that recurring jets may be explained by magnetic reconnection (Shibata et al. 1992; Canfield et al. 1996). In the first and fourth jets, we could see that crossing structures were formed by plasma produced by jet eruption and the filament near the jet base. It seemed that the energy of the plasma was too weak to overcome the coronal loops. Similarly, Yang et al. (2012) presented a blowout surge confined by large coronal loops. In contrast, instead of erupting filament, the filament in our study only had a weak brightening. It is noteworthy that the role of the large coronal loops in our study seemed to confine the ejecting plasma and guide its motion, but the plasmas produced by recurring jets were not along the paths of preexisting loops. We also observed flux emergence at the photospheric magnetic field, and flare-like brightening appeared at the jet base. The collision of the emerging flux and the preexisting magnetic flux system in solar atmosphere leads to the formation of current sheets and field line reconnection ( Moreno-Insertis, F. 2008). The reconnection outflows lead to launching of highspeed and high-temperature plasma. Our observations suggest that the flux, which flowed from the active region, had direct relationship with the recurring jets. It would be interesting to investigate whether other eruptions and their concomitant phenomenon, which were near the active regions, also had flux flowing out of/from the active region.

\section{ACKNOWLEDGMENTS}

The authors thank the anonymous referee for her/his careful reading of the manuscript and constructive comments that improved the original version. The AIA and HMI images used here are courtesy of SDO (NASA) and the AIA/HMI consortia. The $\mathrm{H} \alpha$ image is courtesy by GONG. We thank the AIA, HMI and GONG teams for the easy access to calibrated data. This work is supported by the Natural Science Foundation of China under grant 11163004.

\section{REFERENCES}

Canfield, R. C., Reardon, K. P., \& Leka, K. D. 1996, $\mathrm{H} \alpha$ Surges and X-Ray Jets in AR 7260, ApJ, 464, 1016

Chae, J., Qiu, J., Wang, H., \& Goode, P. R. 1999, Extreme-Ultraviolet Jets and Ha Surges in Solar Microflares, ApJ, 513, L75

Chen, H. D., Jiang, Y. C., \& Ma, S. L. 2009, An EUV Jet and $\mathrm{H} \alpha$ Filament Eruption Associated with Flux Cancelation in a Decaying Active Region, SoPh, 255, 79

Chifor, C., Young, P. R., Isobe, H., Mason, H. E., Tripathi, D., Hara, H., \& Yokovama, T. 2008, An Active Region Jet Observed with Hinode, A\&A, 481, L57

Moreno-Insertis, F. 2008, Magnetic Flux Emergence and Solar Eruptions, IAU, 259

Hong, J. C., Jiang, Y. C., Zheng, R. S, et al. 2011, A Micro Coronal Mass Ejection Associated Blowout Extreme-Ultraviolet Jet, ApJ, 738, L20

Jiang, Y. C., Chen, H. D., Li, K. J., Shen, Y. D., \& Yang, L. H. 2007, The H $\alpha$ Surges and EUV Jets from Magnetic Flux Emergences and Cancellations, A\&A, 469, 331

Jiang, Y. C., Shen, Y., Yi, B., Yang, J., \& Wang, J. 2008, Magnetic Interaction: a Transequatorial Jet and Interconnecting Loops, ApJ, 677, 699

Liu, Y., \& Kurokawa, H. 2004, On a Surge: Properties of an Emerging Flux Region, ApJ, 610, 1136

Lemen, J. R., Title, A. M., Akin, D. J., et al. 2012, The Atmospheric Imaging Assembly (AIA) on the Solar Dynamics Observatory (SDO), SoPh, 275,17L

Pariat, E., Antiochos, S. K., \& DeVore, C. R. 2009, A Model for Solar Polar Jets, ApJ, 691, 61

Pariat, E., Antiochos, S. K., \& DeVore, C. R. 2010, Three-Dimensional Modeling of Quasi-Homologous Solar Jets, ApJ, 714, 1762

Patsourakos, S., Pariat, E., Vourlidas, A., Antiochos, S. K., \& Wuelser, J. P. 2008, STEREO SECCHI Stereoscopic Observations Constraining the Initiation of Polar Coronal Jets, ApJ, 680, L73 
Schou, J., Borrero, J. M., Norton, A. A., et al. 2012, Polarization Calibration of the Helioseismic and Magnetic Imager (HMI) onboard the Solar Dynamics Observatory (SDO), SoPh, 275, 327s

Shen, Y. D., Liu, Y., Su, J. T., \& Deng, Y. Y. 2012, On a Coronal Blowout Jet: the First Observation of a Simultaneously Produced Bubble-Like Cme and a Jet-Like Cme in a Solar Event, ApJ, 745, 164

Shibata, K., Ishido, Y., Acton, L. W., et al. 1992, Observations of X-Ray Jets with the YOHKOH Soft X-Ray Telescope, PASJ, 44, L173

Shimojo, M., Hashimoto, S., Shibata, K., et al. 1996, Statistical Study of Solar X-Ray Jets Observed with the YOHKOH Soft X-Ray Telescope, PASJ, 48, 123

Shimojo, M., Shibata, K., \& Harvey, K. L. 1998, Magnetic Field Properties of Solar X-Ray Jets, SoPh, 178,379

Wang, Y. M., \& Sheeley, N. R. 2002, Coronal WhiteLight Jets near Sunspot Maximum, ApJ, 575, 542

Yang, L. H., Jiang, Y. C., Bi Y., Zheng, R. S., \& Hong, J. C. 2011. Observations of EUV and Soft X-Ray Recurring Jets in an Active Region, RAA, 1229, 1242

Yang, J. Y., Jiang, Y. C., Yang, B., Hong, J. C., Yang, D., Bi, Y., Zheng, R. S., \& Li, H. D. 2012, A Blowout Surge from the Eruption of a Miniature Filament Confined by Large Coronal Loops, NewA, 17, 732 